\title{
The calmodulin-binding transcription activator CAMTAl is required for long-term memory formation in mice
}

\author{
Carlos Bas-Orth, ${ }^{1}$ Yan-Wei Tan, ${ }^{1}$ Ana M.M. Oliveira, ${ }^{1}$ C. Peter Bengtson, \\ and Hilmar Bading \\ Department of Neurobiology, Interdisciplinary Center for Neurosciences, University of Heidelberg, 69120 Heidelberg, Germany
}

\begin{abstract}
The formation of long-term memory requires signaling from the synapse to the nucleus to mediate neuronal activitydependent gene transcription. Synapse-to-nucleus communication is initiated by influx of calcium ions through synaptic NMDA receptors and/or L-type voltage-gated calcium channels and involves the activation of transcription factors by calcium/calmodulin signaling in the nucleus. Recent studies have drawn attention to a new family of transcriptional regulators, the so-called calmodulin-binding transcription activator (CAMTA) proteins. CAMTAs are expressed at particularly high levels in the mouse and human brain, and we reasoned that, as calmodulin-binding transcription factors, CAMTAs may regulate the formation of long-term memory by coupling synaptic activity and calcium/calmodulin signaling to memory-related transcriptional responses. This hypothesis is supported by genetic studies that reported a correlation between Camta gene polymorphisms or mutations and cognitive capability in humans. Here, we show that acute knockdown of CAMTA1, but not CAMTA2, in the hippocampus of adult mice results in impaired performance in two memory tests, contextual fear conditioning and object-place recognition test. Short-term memory and neuronal morphology were not affected by CAMTA knockdown. Gene expression profiling in the hippocampus of control and CAMTA knockdown mice revealed a number of putative CAMTAl target genes related to synaptic transmission and neuronal excitability. Patch clamp recordings in organotypic hippocampal slice cultures provided further evidence for CAMTAl-dependent changes in electrophysiological properties. In summary, our study provides experimental evidence that confirms previous human genetic studies and establishes CAMTAl as a regulator of long-term memory formation.
\end{abstract}

[Supplemental material is available for this article.]

The formation of long-term memory depends on the ability of neurons to undergo long-lasting changes in their functional and morphological properties. These adaptive responses require signaling from the synapse to the nucleus to mediate neuronal activity-dependent gene transcription. Synapse-to-nucleus communication is initiated by influx of calcium ions through synaptic NMDA receptors and/or L-type voltage-gated calcium channels and involves the activation of transcription factors by calcium/ calmodulin $\left(\mathrm{Ca}^{2+} / \mathrm{CaM}\right)$ signaling in the nucleus (Bading et al. 1993; Limback-Stokin et al. 2004; West and Greenberg 2011; Bading 2013). Several calcium- and activity-dependent transcriptional regulators with roles in memory formation have been identified in neurons, which include CREB, CBP, SRF, MeCP2, MEF2, and DREAM (Mellstrom et al. 2008; Alberini 2009). Recent studies have drawn attention to a new family of transcriptional regulators, the so-called calmodulin-binding transcription activator (CAMTA) proteins (Bouché et al. 2002; Yang and Poovaiah 2002; Finkler et al. 2007). CAMTA proteins are present in a wide range of organisms from simple unicellular eukaryotes to plants and animals and are involved in a range of adaptive signaling events that include plant stress responses (Finkler et al. 2007), adaptation of phototransduction in flies (Han et al. 2006), and regulation of cardiac hypertrophy in mice (Song et al. 2006).

\footnotetext{
'These authors contributed equally to this work. Corresponding author: Hilmar.Bading@uni-hd.de Article is online at http://www.learnmem.org/cgi/doi/10.1101/lm.041111. 115 .
}

Here, we tested the hypothesis that CAMTAs may play a role in long-term memory based on the following reasons: First, as calmodulin-binding transcriptional activators CAMTA proteins may couple synaptic activity and $\mathrm{Ca}^{2+} / \mathrm{CaM}$ signaling to memory-related transcriptional responses. Second, CAMTAs are expressed at particularly high levels in the mouse and human brain (Huentelman et al. 2007; Lein et al. 2007). Third, several genetic studies reported a link between Camta gene polymorphisms or mutations and cognitive capability in humans (Huentelman et al. 2007; Miller et al. 2011; Thevenon et al. 2012).

\section{Results}

Expression of CAMTA proteins during postnatal development and adulthood

In situ hybridization studies (Lein et al. 2007) indicate that Camta mRNAs are highly expressed in the hippocampus. Available data for CAMTA protein expression in the brain are, however, very limited (Huentelman et al. 2007; Uhlen et al. 2010). We thus performed immunoblot analyses of CAMTA protein expression in hippocampal tissue harvested from mice at different

(C) 2016 Bas-Orth et al. This article is distributed exclusively by Cold Spring Harbor Laboratory Press for the first 12 months after the full-issue publication date (see http://learnmem.cshlp.org/site/misc/terms.xhtml). After 12 months, it is available under a Creative Commons License (AttributionNonCommercial 4.0 International), as described at http://creativecommons. org/licenses/by-nc/4.0/. 


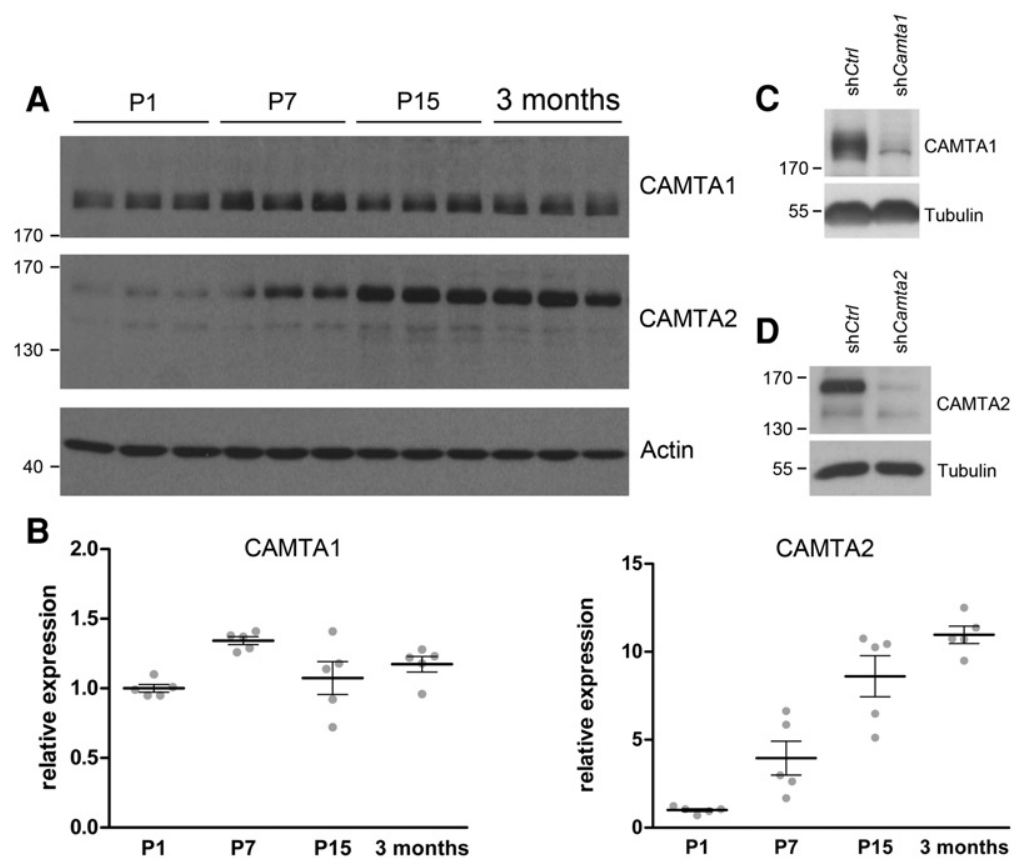

Figure 1. Analysis of CAMTA protein expression in mouse hippocampus. $(A)$ Representative immunoblot showing expression of CAMTA1, CAMTA2, and $\beta$-Actin in mouse hippocampus at postnatal day (P) 1,7 , and 15 , and at 3 mo. Position of molecular weight markers $(\mathrm{kDa})$ is indicated. (B) Quantification of CAMTA1 and CAMTA2 protein expression (normalized to $\beta$-actin). Mean \pm SEM and individual values are shown. $N=5$ animals per group. $(C, D)$ Specificity of CAMTA1 $(C)$ and CAMTA2 $(D)$ antibodies was confirmed by immunoblot analysis of primary mouse hippocampal neurons infected with control or Camta1 shRNA (C), or control or Camta2 shRNA (D).

developmental stages (Fig. 1A,B). Antibody specificity was confirmed using Camta1- or Camta2-specific shRNAs (Fig. 1C,D).

CAMTA1 was consistently expressed throughout postnatal development and adulthood with a small peak around postnatal day (P) 7. In contrast, CAMTA2 expression was hardly detectable at P1 but increased eightfold during the first two postnatal weeks. In young adult mice CAMTA2 expression was 11-fold compared with P1. The sharp increase in CAMTA2 expression between P1 and P15, a time that is marked by increased synapse formation and the maturation of neuronal circuits, suggests that CAMTA2 might indeed play a role in cognitive functions.

\section{CAMTA1, but not CAMTA2, is required for long-term memory formation}

We next tested the role of CAMTAs in memory formation. The two mouse Camta genes (Camta1 and Camta2) are highly homologous and might compensate for each other in loss-of-function experiments. We therefore generated a set of recombinant adeno-associated viruses (rAAV) that express shRNAs targeting Camta1, Camta2, or both. To better control for potential off-target effects, we generated two different shRNA constructs for each Camta gene (see Materials and Methods for details). We confirmed the efficiency and specificity of the shRNA constructs by immunoblot analysis of primary mouse hippocampal neurons infected with the respective viruses (Fig. 2A). To test whether CAMTA proteins are generally required for long-term memory we knocked down both genes simultaneously in the dorsal hippocampus of adult mice. This was achieved by stereotaxic delivery of a rAAV that expresses two shRNAs, targeting Camta1 and Camta2, respectively. Expression of the two shRNA sequences in vivo significantly reduced the endogenous expression of Camta1 and 2 compared with the expression of a control shRNA (shCtrl2x)
(Fig. 2B). We next assessed the effect of Camta 1 and 2 knockdown on cognitive abilities. Behavioral analysis using the hippocampus-dependent test, contextual fear conditioning revealed that when mice were re-exposed to the conditioned stimulus (context) $24 \mathrm{~h}$ after receiving the foot shock, the shCamta1+2 group spent significantly less time freezing compared with the shCtrl2x group. shCtrl $2 x$ - and shCamta1+2-injected mice did not differ in overall activity during the training session (Table 1) indicating normal basal locomotor activity. When testing occurred $1 \mathrm{~h}$ after conditioning, the two groups did not differ in the percentage of freezing (Fig. 2C). This indicates a specific role for CAMTA 1 and/or 2 in long-term memory formation. We further confirmed the effect on hippocampal long-term memory using the object-place recognition test. This test relies on the ability of mice to recognize a change in an object's location and is dependent on hippocampal function. During a first exposure to the objects (training session), all mice showed equal exploration of the object to be displaced showing no natural preference for that object. Also, hippocampal Camta1 and 2 double knockdown did not interfere with the total time that the mice spent exploring the objects (Table 1 ). When tested $24 \mathrm{~h}$ after training, the shCamta $1+2$-injected mice explored less the displaced object compared with the shCtrl2x group (Fig. 2D). This reduced preference for the displaced object suggests impaired long-term memory for the object's spatial location.

To test whether CAMTA1, CAMTA2, or both are required for long-term memory formation we knocked down each gene individually in the dorsal hippocampus of adult mice. Given the sharp increase in hippocampal CAMTA2 expression during postnatal development, CAMTA2 appeared like a good candidate for playing a role in memory formation in the adult. To investigate this possibility, we designed two distinct Camta2-specific shRNAs (shCamta2A and shCamta2B). The expression of either one in the mouse hippocampus significantly knocked down endogenous Camta2 expression (mean $\pm \mathrm{SEM}, 1.00 \pm 0.05$ in shCtrl versus $0.38 \pm 0.01$ in shCamta $2 A, P=0.00002$ versus $0.32 \pm 0.02$ in shCamta2B, $P=0.00001 ; P$-values determined by Tukey's multiple comparison test), without affecting Camta1 (mean \pm SEM, $1.00 \pm 0.06$ in shCtrl versus $1.21 \pm 0.11$ in shCamta2A, $P=0.226$ versus $0.96 \pm 0.07$ in shCamta2B, $P=0.926 ; P$-values determined by Tukey's multiple comparison test). Behavioral analysis of mice expressing shCtrl, shCamta2A or shCamta2B showed that all groups expressed similar freezing percentage in a 24 -h test in contextual fear conditioning (mean \pm SEM, 36.82 \pm 3.73 in shCtrl versus $38.51 \pm 4.54$ in shCamta2A, $P=0.946$ versus $30.67 \pm 2.94$ in shCamta2B, $P=0.461$; $P$-values determined by Tukey's multiple comparison test). These findings were unexpected as they indicate that CAMTA2 loss-of-function has no effect on adult hippocampal long-term memory. We next tested whether Camta2 knockdown has an effect on memory persistence and tested shCtrl-, shCamta2A-, or shCamta2B-injected mice in contextual fear conditioning $1 \mathrm{wk}$ after conditioning. Again, no difference between the groups was observed (mean \pm SEM, $47.7 \pm 4.6$ in 
A

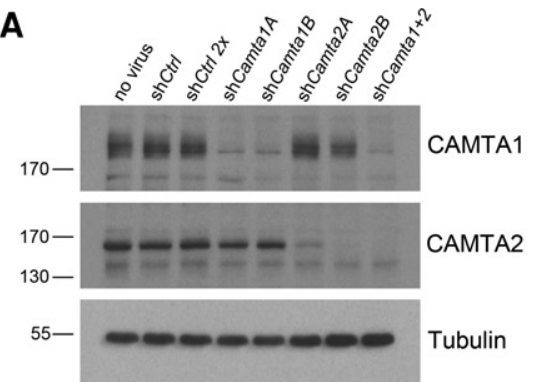

B

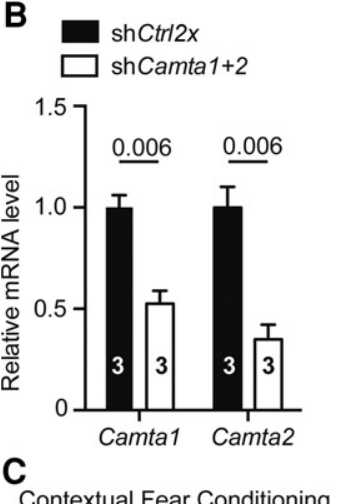

Contextual Fear Conditioning

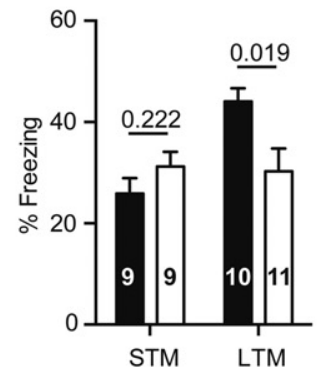

D

Object-place Recognition

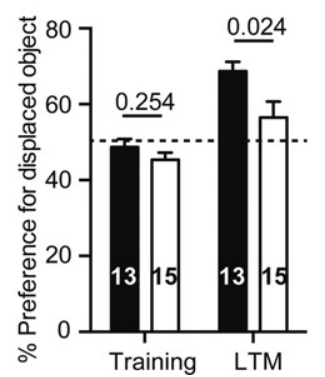

E

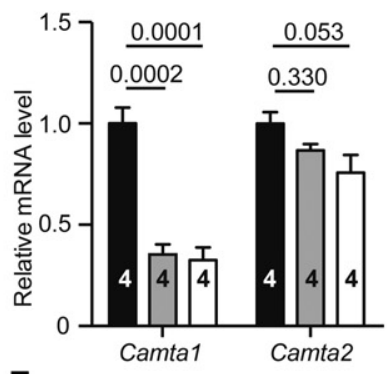

$\mathbf{F}$

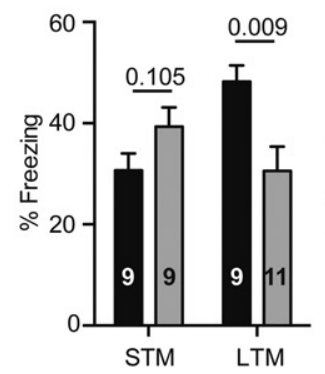

G

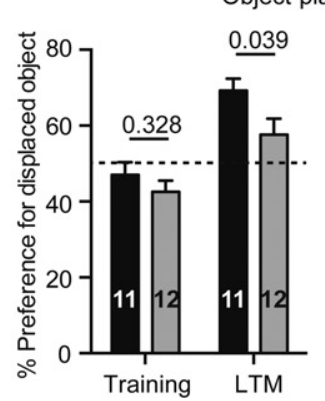

We next tested the effect of hippocampal Camta1 knockdown on memory. Two distinct Camta1-specific shRNA sequences (shCamta1A and shCamta1B) were used. Expression of either shCamta1A or shCamta1B in the adult mouse hippocampus efficiently knocked down endogenous Camta1 without affecting Camta2 (Fig. 2E; Supplemental Fig. 1). Knockdown of hippocampal Camta1 using shRNA sequences A or B significantly impaired long-term contextual fear memory (Fig. 2F). In contrast, the ability to form short-term memories after contextual fear conditioning was not different between the control group (shCtrl) and shCamta1A or shCamta1B groups. A similar impairment in long-term memory formation was observed using the object-place recognition test. In this test, the control group of animals (shCtrl) but not the mice expressing shCamta1A or shCamta1B showed the typical increased preference for the displaced object $24 \mathrm{~h}$ after training (Fig. 2G). No change was observed in motor or exploratory activity and shock response in mice injected with shCtrl, shCamta1A, or shCamta1B (Table 1). Together, these data demonstrate a critical role for CAMTA1 expression in the hippocampus in the formation of long-term memory. Moreover, these memory deficits in Camta1 knockdown mice are not compensated for by CAMTA2 expression, which itself is unaffected by Camta1 knockdown, indicating that the functions of CAMTA1 and CAMTA2 are not redundant.

\section{CAMTA loss-of-function does not} affect neuronal morphology in the adult hippocampus

To obtain insight into the mechanisms through which CAMTA1 may influence long-term memory formation, we explored a possible effect on neuronal morphology, in particular dendritic structure. A role for CAMTA1 for the structural integrity of neurons has recently been shown for Purkinje cells in the cerebellum (Long et al. 2014). Mice lacking the Camta1 gene suffer from progressive postnatal neuronal atrophy in the cerebellum leading to motor deficits and ataxia (Long et al. 2014). Furthermore, ectopic expression of CAMTA1 in a neuroblastoma cell line led to the formation of neurite-like processes in these cells (Henrich et al. 2011). These findings are consistent with a role of CAMTA1 in the formation and/or maintenance of

shCtrl versus $39.3 \pm 3.8$ in shCamta $2 A, P=0.436$ versus $45.8 \pm 5.7$ in shCamta $2 B, P=0.956 ; P$-values determined by Tukey's multiple comparison test). dendrites and raise the possibility that CAMTA loss-of-function could result in reduced dendritic complexity, an effect that could explain the observed deficits in memory formation. To exclude 
Table 1. Exploratory and locomotor activity of mice injected with shCtrl2x, shCamta1+2, shCtrl, shCamta1A, or shCamta1B

\begin{tabular}{|c|c|c|c|c|c|c|}
\hline & \multirow{2}{*}{\multicolumn{2}{|c|}{$\begin{array}{c}\begin{array}{c}\text { Object-place } \\
\text { recognition test }\end{array} \\
\begin{array}{c}\text { Total object } \\
\text { exploration (sec) }\end{array}\end{array}$}} & \multicolumn{4}{|c|}{ Contextual fear conditioning } \\
\hline & & & \multicolumn{2}{|c|}{$\begin{array}{l}\text { Moving speed before } \\
\text { shock }(\mathrm{cm} / \mathrm{sec})\end{array}$} & \multicolumn{2}{|c|}{$\begin{array}{l}\text { Moving speed during } \\
\text { shock }(\mathrm{cm} / \mathrm{sec})\end{array}$} \\
\hline & Mean \pm SEM & $P$ value & Mean \pm SEM & $P$ value & Mean \pm SEM & $P$ value \\
\hline $\operatorname{shCtrl2x}$ & $34.0 \pm 2.17$ & 0.06 & $9.47 \pm 0.71$ & 0.63 & $32.89 \pm 2.16$ & 0.92 \\
\hline shCamta1+2 & $27.39 \pm 2.60$ & & $10.45 \pm 0.65$ & & $33.20 \pm 2.35$ & \\
\hline shCtrl & $31.65 \pm 4.60$ & 0.95 & $10.65 \pm 0.69$ & 0.14 & $38.36 \pm 1.86$ & 0.11 \\
\hline shCamta1A & $32.08 \pm 5.40$ & & $11.85 \pm 0.41$ & & $42.94 \pm 2.07$ & \\
\hline shCtrl & $37.84 \pm 5.88$ & 0.39 & $11.33 \pm 0.58$ & 0.14 & $38.53 \pm 1.56$ & 0.27 \\
\hline shCamta1B & $31.51 \pm 4.01$ & & $12.38 \pm 0.39$ & & $41.88 \pm 2.52$ & \\
\hline
\end{tabular}

The parameters were analyzed during the object-place recognition test and fear conditioning training sessions. $P$-values were determined by two-tailed $t$-test.

any form of compensation, we focused our analysis on Camta $1+2$ double knockdown infected animals. Within 1-2 wk after the completion of behavioral testing we subjected the brains of control (shCtrl2x) and Camta knockdown animals to Golgi impregnation and analyzed the morphology of basal dendrites of hippocampal CA1 pyramidal neurons (Fig. 3). We found that dendritic length and complexity, as well as dendritic spine density and length were not significantly different between control and Camta knockdown animals. To exclude a potential confounding effect of the Camta1 +2 double knockdown and to specifically test for a potential role of CAMTA1 in the regulation of dendritic morphology, we repeated the experiment with an independent cohort of animals injected with control virus (shCtrl) or Camta1 knockdown virus (shCamta1A). Again, we did not find a difference between control and Camta knockdown animals (Fig. 3). Thus CAMTA1 affects long-term memory formation via a mechanism that does not involve the regulation of neuronal morphology in the adult hippocampus.

\section{CAMTA1 loss-of-function does not broadly affect gene transcription in the adult hippocampus}

Given the well-described role of CAMTA1 as a transcriptional activator, we next aimed to identify genes that are regulated by CAMTA1 in the adult mouse hippocampus. We used RNA-seq to analyze the gene expression profile of hippocampal tissue from mice that had been infected with rAAVs expressing either shCamta1A, shCamta1B, or shCtrl. RNA was isolated from mCherry-positive rAAV-infected parts of the dorsal hippocampus. Thus, the hippocampal region used for RNA-seq corresponds to the region affected by Camta knockdown in the behavioral experiments. Complete RNA-seq data and gene lists have been deposited in NCBI's Gene Expression Omnibus and are accessible through GEO Series accession number GSE62122.

We reasoned that CAMTA1 may regulate either the basal expression of genes (i.e., genes that are not regulated by neuronal activity or genes that are driven by basal levels of neuronal activity), or learning-induced gene transcription. To maximize the potential to detect CAMTA1-regulated genes while minimizing the number of animals used for the RNA-seq screen, we examined gene expression levels in the hippocampus of Camta1 knockdown and control mice following object-place recognition learning (see Materials and Methods for details). We reasoned that, if CAMTA1 specifically regulates an activity-dependent change in expression of a given gene, the levels of this gene would be different between shCtrl- and shCamta1-infected animals after training regardless of its basal expression level. Likewise, if CAMTA1 regulates the basal expression of an activity-indepen- dent gene, differences between shCtrland shCamta1-infected animals would still be detectable after training.

Unexpectedly, the RNA-seq analysis did not reveal major changes in gene expression between shCtrl- and shCamta1-infected animals. Based on typical threshold criteria (adjusted $P$ value $<0.05$; up- or downregulation greater than twofold), we found only 29 genes that were differentially expressed between shCtrl- and shCamta1infected groups (Supplemental Table 1, see also Gene Expression Omnibus series GSE62122 for complete gene lists). Most of these genes were related to immune system function. To identify potential CAMTA1 target genes for further analysis, we selected all genes from the RNA-seq data that showed an up- or down-regulation of more than 1.2-fold with both Camta1 shRNAs, and that had an adjusted $P$-value $<0.1$. Application of this relaxed threshold criteria resulted in a set of 193 candidate genes.

Gene ontology analysis of this gene set revealed an enrichment of several pathways, consistent with diverse CAMTA functions that have been reported in the literature (Finkler et al. 2007). Major enriched pathways were related to immune system function $\left(P=1 \times 10^{-13}\right)$, cell adhesion $\left(P=1.47 \times 10^{-05}\right)$, programmed cell death $(P=0.01)$, cancer $(P=0.03)$, and calcium signaling $(P=0.08)$. In addition, manual annotation of the gene list revealed that 24 of the 193 genes encode for ion channels, neurotransmitter receptors, or structural proteins that have been related to synaptic transmission, synaptic structure, or neuronal excitability (see Table 2 for examples, Supplemental Table 2 for complete list). Thus, the RNA-seq analysis did not reveal major changes in global gene expression or changes in the expression of well-known plasticity genes, but suggested a set of potential CAMTA1 target genes with neuronal functions.

To validate potential CAMTA1 target genes from the RNA-seq screen, we selected a set of 10 genes from Table 2 for QRT-PCR analysis of hippocampal tissue obtained from an independent and larger cohort of mice that again were injected with rAAVs expressing either shCamta1A, shCamta1B, or shCtrl (Fig. 4). To test whether Camta1 knockdown affects basal or activity-regulated transcription of the selected genes, we this time also included an untrained group of animals (which had remained in the home cage) and compared gene expression in untrained animals to animals that were trained in the object-place recognition test. To confirm that the object-place recognition training had successfully induced a genomic response we first analyzed the expression of four well-characterized activity-regulated genes, i.e., Arc, Npas4, Gadd45b, and cFos (Fig. 4A). At the time point used for our analysis (90 min after the start of training, see Materials and Methods for details), we detected a training-mediated induction of $A r c$, Gadd $45 b$ and $c F o s$, but not of Npas4. Overall, basal levels of gene expression as well as training-mediated gene induction were similar between shCtrl, shCamta1A, and shCamta1B injected animals. Induction of $G a d d 45 b$ was reduced slightly, but statistically not significantly, by Camta1 knockdown (mean difference between trained and basal $=0.31,95 \% \mathrm{CI}=0.031-0.595$ for shCtrl; 0.17 , -0.191 to 0.523 for shCamta $1 A ; 0.09,-0.261$ to 0.431 for shCamta1B). These data indicate that CAMTA1 loss-of-function does not generally affect learning-induced gene transcription. We next analyzed the expression of our set of 10 candidate genes (Atp2b4, Cnr1, Doc2g, Htr2c, Kcnd3, Otof, Scn7a, Sytl4, Tgfb2, Trpc7) that we had selected from the list of genes related to neuronal function (Table 2; Supplemental Table 2). We found that 
A

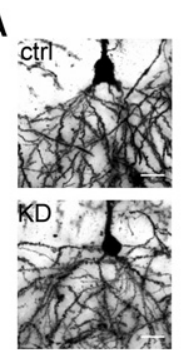

B

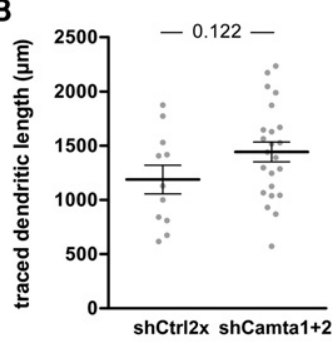

D

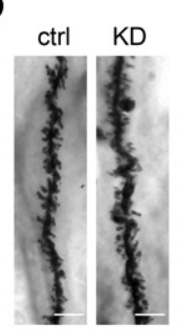

E

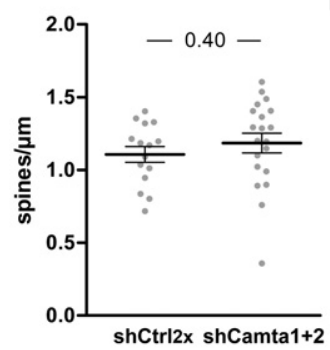

C

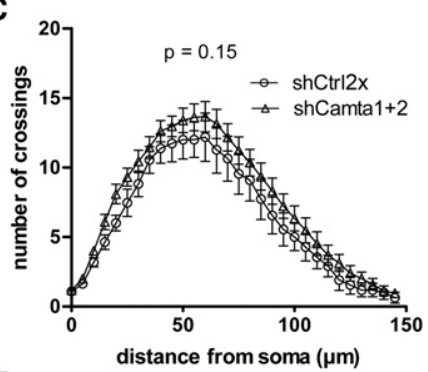

$F$

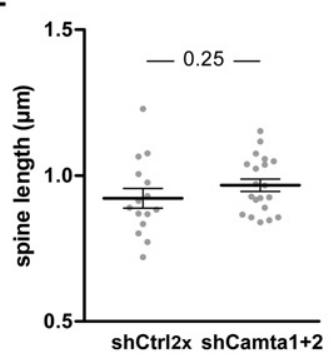

G

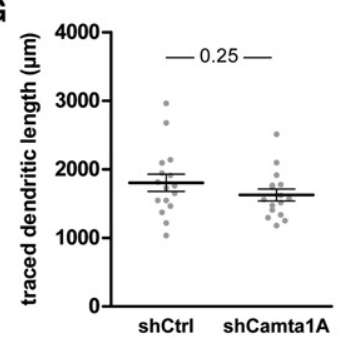

I

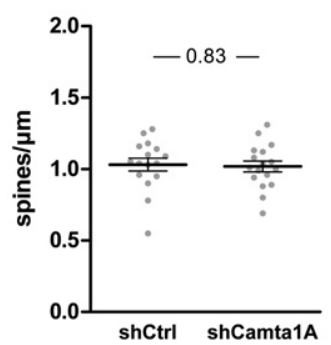

H

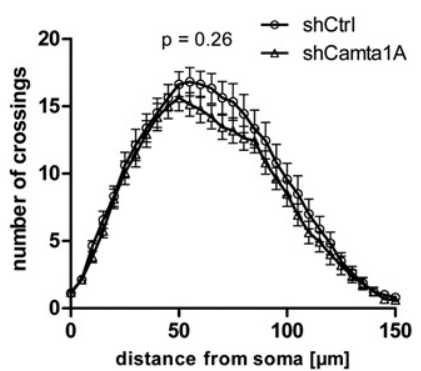

J

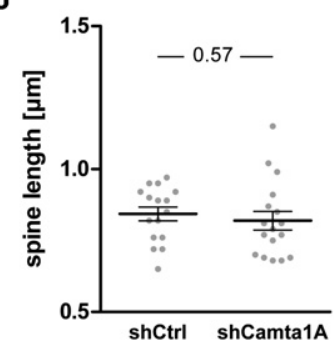

Figure 3. Acute Camta1 knockdown does not affect neuronal morphology. The effect of Camta1+2 double knockdown $(A-F)$, and Camta1 single knockdown $(G-J)$ on CA1 pyramidal neuron morphology was assessed. $(A)$ Representative photomicrographs of Golgi-impregnated CA1 pyramidal neurons. Focused projections of 3-D image stacks are shown. Scale bar, $20 \mu \mathrm{m}$. (B) Quantification of traced dendritic length of basal dendrites. Mean \pm SEM and individual values are shown for $n=11$ neurons from three animals (sh $C$ trl $2 x)$ and $n=23$ neurons from five animals (shCamta $1+2)$. (C) Sholl analysis of traced basal dendrites. Mean \pm SEM for $n=11$ neurons from three animals (shCtrl2x) and $n=23$ neurons from five animals (shCamta1+2) are shown. (D) Representative photomicrographs of Golgi-impregnated segments of CA1 pyramidal neuron basal dendrites. Focused projections of 3-D image stacks are shown. Scale bar, $5 \mu \mathrm{m}$. $(E, F)$ Quantification of spine density $(E)$ and spine length $(F)$. Mean \pm SEM and individual values are shown for $n=15$ dendritic segments from three animals (shCtrl2x) and $n=20$ dendritic segments from four animals (shCamta1 +2 ). (G) Quantification of traced dendritic length of CA1 pyramidal neuron basal dendrites. Mean \pm SEM and individual values are shown for $n=16$ neurons from five animals per group. $(H)$ Sholl analysis of traced basal dendrites. Mean \pm SEM for $n=16$ neurons from five animals per group are shown. $(I, J)$ Quantification of spine density $(I)$ and spine length $(J)$. Mean \pm SEM and individual values are shown for $n=16$ dendritic segments from five animals (sh Ctrl) and $n=17$ dendritic segments from five animals (sh Camta1A). P-values were determined by two-tailed t-test (traced dendritic length, spine density, and spine length) or repeated-measures two-way ANOVA with Bonferroni post hoc test (Sholl analysis of dendritic complexity). Ctrl, shCtrl2x; KD, shCamta1+2.

training had no effect on the expression of this gene set (Fig. 4B). This result is in line with previous data showing that none of these genes belong to the group of classical activity-regulated genes
(Zhang et al. 2007, 2009). When assessing the effect of Camta1 knockdown, we only found small, nonsignificant differences between shCtrl, shCamta1A, and shCamta1B injected animals on these genes (Fig. 4B). Together, the results from the RNA-seq and QRT-PCR studies indicate that CAMTA1 loss-of-function in the adult hippocampus does not impair learning-induced gene transcription and has only moderate effects on the cells' gene expression profile.

\section{CAMTA1 knockdown affects electrophysiological properties associated with the excitability and synaptic transmission in CAl pyramidal neurons}

We performed whole cell patch clamp recordings of CA1 pyramidal neurons in organotypic hippocampal slice cultures to test whether the observed changes in gene expression correlate with changes in electrophysiological properties of neurons (Table 3). Slice cultures were infected with rAAV-shCtrl or rAAV-shCamta1B on DIV 5 and were used for patch clamp recordings on DIV 28-35. Efficiency of Camta1 knockdown in rat neurons was verified by Immunoblot analysis of primary rat hippocampal neurons (Fig. 5C) and rat organotypic cultures (Fig. 5D) that had been infected with rAAV-shCtrl or rAAV-shCamta1B. Camta1 knockdown did not affect whole-cell membrane capacitance or resistance, action potential threshold, amplitude or half width, or hyperpolarization-activated conductances (h current or fast inward rectifier). We found, however, that knockdown of Camta1 affected factors associated with electrical excitability. In comparison to shCtrl-infected cells, shCamta1B infected neurons were slightly more hyperpolarized and showed an increased amplitude of the afterhyperpolarization potential (AHP) without significantly affecting its delay. Despite this, the action potential profile evoked by current injection was not altered in shCamta1B infected neurons which did not differ from control neurons in the accommodation of the firing rate over a 1 -sec depolarizing step. Knockdown of Camta1 expression reduced the amplitude and increased the inter-event interval of miniature post-synaptic currents (mEPSCs) consistent with reduced synaptic transmission. 
Table 2. Examples of CAMTA1-regulated genes with known neuronal function

\begin{tabular}{|c|c|c|c|}
\hline $\begin{array}{l}\text { Gene } \\
\text { symbol }\end{array}$ & Description & $\begin{array}{c}\mathrm{FC} \\
\operatorname{sh} A\end{array}$ & $\begin{array}{c}\text { FC } \\
\operatorname{sh} B\end{array}$ \\
\hline Camta1 & Calmodulin binding transcription activator 1 & 0.50 & 0.49 \\
\hline Camta2 & Calmodulin binding transcription activator 2 & 1.03 & 1.02 \\
\hline Atp2b4 & $\mathrm{Ca}^{2+}$ transporting ATPase & 0.75 & 0.67 \\
\hline Kond3 & Voltage-gated potassium channel & 0.74 & 0.83 \\
\hline Kcnj16 & Inwardly rectifying potassium channel & 0.75 & 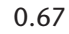 \\
\hline Scn7a & Voltage-gated sodium channel & 0.34 & 0.67 \\
\hline Trpc7 & Transient receptor potential cation channel & 0.44 & 0.53 \\
\hline Syt14 & Synaptotagmin-like 4 & 0.44 & 0.62 \\
\hline Otof & Otoferlin & 0.55 & 0.40 \\
\hline Doc2g & Double $\mathrm{C} 2 \gamma$ & 1.20 & 1.73 \\
\hline $\mathrm{Htr} 2 \mathrm{c}$ & 5-Hydroxytryptamine (serotonin) receptor $2 \mathrm{C}$ & 0.52 & 0.35 \\
\hline Cnr1 & Cannabinoid receptor 1 & 0.80 & 0.81 \\
\hline Tgfb2 & Transforming growth factor $\beta 2$ & 0.78 & 0.80 \\
\hline
\end{tabular}

From the 193 putative CAMTA1 target genes identified in this study, 24 are known to regulate neuronal function. This table lists gene symbol, name, and relative expression for selected examples. Relative mRNA expression levels of Camta1 and Camta2 indicate efficient and specific knockdown of Camta1. For complete gene lists see NCBI Gene Expression Omnibus GSE62122. (FC) fold change relative to shCtrl.

combination of them in the hippocampus of adult mice we found that (i) CAMTA1, but not CAMTA2, is required for long-term memory formation, (ii) CAMTAs are not required for short-term memory formation or the maintenance of neuronal morphology, (iii) CAMTA1 loss-of-function has only moderate effects on hippocampal gene expression, and (iv) CAMTA1 loss-of-function affects electrical excitability.

These results are in line with and extend previous genetic studies in humans that revealed a statistical correlation between specific Camta1 single nucleotide polymorphisms (SNPs) or intragenic Camta1 rearrangements and cognitive performance (Huentelman et al. 2007; Miller et al. 2011; Thevenon et al 2012). All identified SNPs were located within intronic regions of the Camta1 gene, leaving unanswered exactly how these polymorphisms affect CAMTA1 function.

In contrast to CAMTA1, the function of CAMTA2 remains unclear. The strong postnatal increase in hippocampal expression of CAMTA2 argues for a role of CAMTA2 in adult brain function. Surprisingly, however, we did not observe any effect of CAMTA2 loss-of-function on long-term memory, neuronal morphology, or survival. Likewise, it was somewhat unexpected that endogenous expression of CAMTA2 was not sufficient to compensate for CAMTA1 loss-of-function, despite the high degree of homology between the two genes. These findings suggest that CAMTA1 and CAMTA2 may have different genomic targets and, consequently, serve distinct functions.

This study was based on the hypothesis that CAMTAs, as transcriptional activators, regulate learning-induced gene transcription to control long-term memory formation. In line with this, our behavioral experiments provide robust evidence for a role of CAMTA1 in long-term memory formation. However, despite comprehensive morphological, electrophysiological, and transcriptomic analyses we could not identify a straightforward mechanism that explains how exactly CAMTA1 affects longterm memory. Our gene profiling experiments revealed a small number of potential target genes that were moderately affected by CAMTA1 loss-of-function. These genes were selected based on a rather low cut-off of 1.20 -fold for differential expression to avoid excluding relevant CAMTA1 target genes. However, this criterion had to be met by two independent shRNAs, which increases the stringency of the selection procedure. We would also like to note that the hippocampal tissue analyzed in our experiments contains many non-neuronal cells, and, moreover, within the densely packed primary neuron cell layers, not all neurons get infected by stereotaxic delivery of the rAAVs. Thus, the measured changes in gene expression are likely to underestimate the changes taking place in individual infected neurons. Accordingly, the reason for the requirement of CAMTA1 in memory consolidation may lie in its ability to regulate a set of functionally related genes that control synaptic transmission and neuronal excitability. This idea is supported by our patch clamp experiments that revealed reduced neuronal excitability and basal synaptic activity upon Camta1 knockdown in organotypic hippocampal slice cultures. Nevertheless, whether the sum of small gene expression changes is indeed responsible for the long-term memory deficit remains an open question. In fact, the rather moderate transcriptional changes may also indicate that CAMTA1 affects long-term memory independent of gene expression, suggesting additional functions of CAMTA1 beyond its well-described role as transcriptional activator.

In summary, polymorphisms and mutations of the Camta1 gene have been correlated with cognitive performance levels in humans. Our experiments in mice now establish CAMTA1 as a novel regulator of long-term memory formation. The mechanisms of CAMTA1 function in memory, however, remain an important question for future studies.

\section{Materials and Methods}

Mice

C57BL/6NCrl mice (Charles River, http://www.criver.com/ products-services/basic-research/find-a-model/c57bl-6n-mouse) were used in this study. Mice were group-housed on a $12 \mathrm{~h}$ lightdark cycle and had ad libitum access to water and food. All procedures were done in accordance with German guidelines for the care and use of laboratory animals and with the European Community Council Directive 86/609/EEC. Experiments were approved by local authorities.

\section{Cell culture}

Primary dissociated cultures of hippocampal neurons from newborn C57BL/6NCrl mice (Charles River) were prepared and maintained as described (Bading and Greenberg 1991; Zhang et al. 2007).

\section{Organotypic slice cultures}

Organotypic hippocampal slices cultures (OTC) were prepared from P7 Sprague Dawley rat pups (Charles River, http://www .criver.com/products-services/basic-research/find-a-model/cdigs-rat) with a McIlwain tissue chopper (Campden Instruments) as previously described (Stoppini et al. 1991). The 400- $\mu \mathrm{m}$ thick slices were prepared in preparation medium and plated on cell culture inserts (Millipore) in contact with incubation medium which was changed every $2-3 \mathrm{~d}$ and kept at $35^{\circ} \mathrm{C}$ in $5 \% \mathrm{CO}_{2}$. OTC preparation medium: $\mathrm{mL}$ for $100 \mathrm{~mL}$ : $94.1 \mathrm{~mL} 1 \times$ MEM, $1 \mathrm{~mL} 1 \times$ Glutamax, $1 \mathrm{~mL} 2.5 \mathrm{M}$ glucose, $2.5 \mathrm{~mL} 1 \mathrm{M}$ HEPES, $1 \mathrm{~mL}$ Pen/Strep or $0.5 \mathrm{~mL}$ Primocin, $0.4 \mathrm{~mL} 1 \mathrm{M} \mathrm{NaOH}$; $\mathrm{pH}$ 7.3-7.4, sterile filtration. OTC incubation medium: $\mathrm{mL}$ for $50 \mathrm{~mL}: 25.375 \mathrm{~mL} 1 \times$ MEM, $12.5 \mathrm{~mL} 1 \times$ BME, $12.5 \mathrm{~mL} 1 \times$ horse serum, $0.5 \mathrm{~mL} 1 \times$ Glutamax, $0.75 \mathrm{~mL} 45 \%$ glucose, $1 \mathrm{M}$ HEPES, $0.5 \mathrm{~mL}$ Pen/Strep or $0.2 \mathrm{~mL}$ Pri-

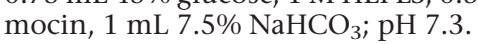

\section{Protein expression analysis}

For protein expression analysis 1-, 7-, and 15-d old male and female mice and 3-mo old male mice were used. No difference in protein expression was observed between male and female animals. Immunoblotting was performed according to 
A

- shCtrl basal (6) $\quad$ shCamta1A basal (5) $\quad$ shCamta1B basal (6)

- shCtrl trained (6) - shCamta1A trained (4) - shCamta1B trained (5)

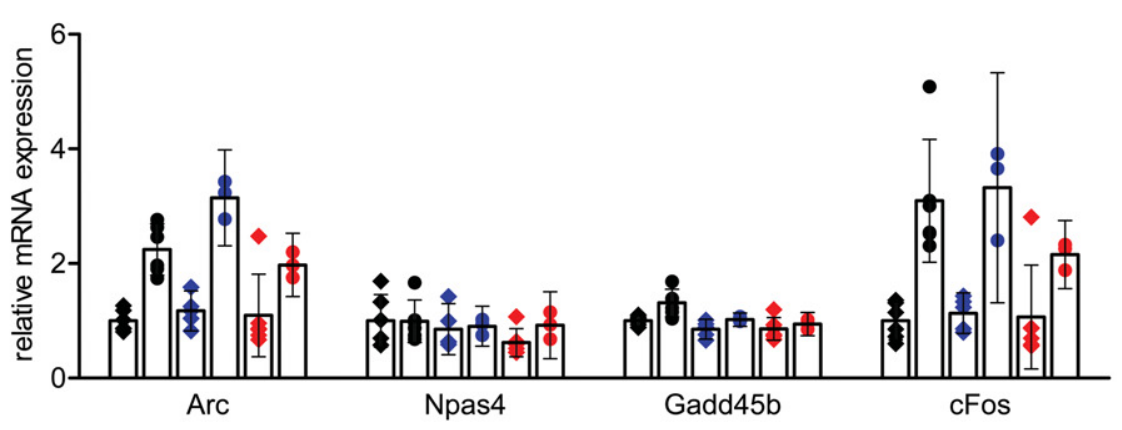

B
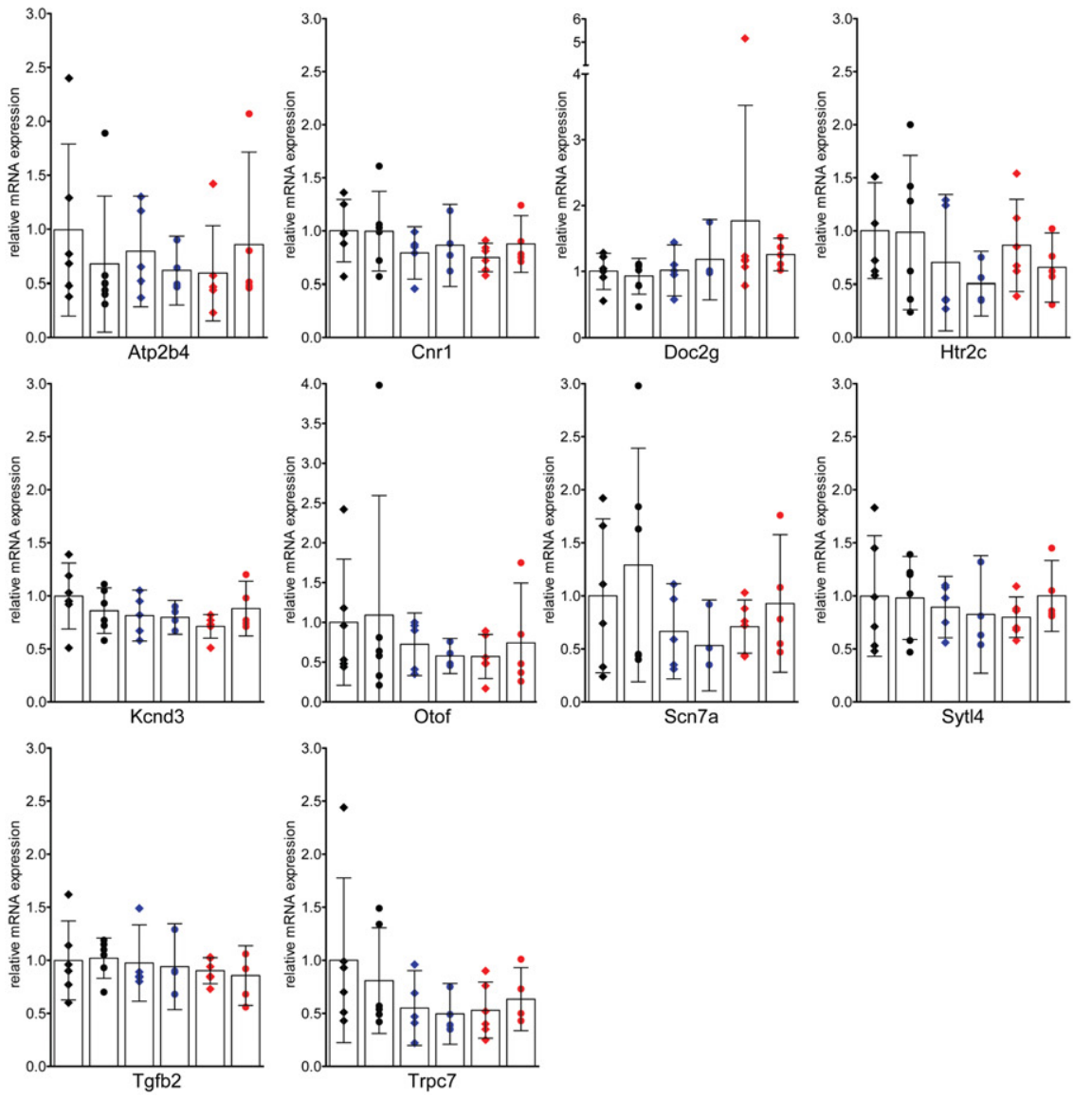

Figure 4. QRT-PCR validation of putative CAMTA1 target genes. (A) Relative mRNA expression of known activity-regulated genes in naïve animals (basal) and in animals that underwent object-place recognition training (trained). Hippocampal tissue was obtained from mice infected with shCtrl, shCamta1A, or shCamta1B. Number of animals per group is indicated in the legend. (B) Relative mRNA expression of indicated genes in the same animals as in $A$. Mean $+95 \% \mathrm{Cl}$, as well as individual values are indicated.

standard procedures. Antibodies were mouse anti- $\beta$-Actin (Santa Cruz sc-47778, 1:1,000), mouse anti- $\alpha$-Tubulin (Sigma T9026, 1:400,000), rabbit anti-CAMTA1 (Sigma HPA036343, 1:500), rabbit anti-CAMTA2 (Abnova PAB15828, 1:1,000), goat anti-mouse HRP and goat anti-rabbit HRP (Dianova, 1:5,000). Enhanced chemiluminescence signals were quantified with ImageJ (http:// imagej.nih.gov/ij/).

\section{Recombinant adeno-associated viruses (rAAVs)}

For the expression of short hairpin RNAs (shRNAs), a rAAV vector was used that contains a U6 promoter for shRNA expression and a CaMKII promoter driving mCherry expression (Lau and Bading 2009). The following shRNA sequences were used $\left(5^{\prime}-3^{\prime}\right)$ : nontargeting control shRNA: GTGCCAAGACGGGTAGTCA (Mauceri et al. 2011), shCamta1A: GG AGCTACTGTATTTAGAC, shCamta1B: AGAGGCAGAGTAGCATTAC, shCamta2A: GCCCCCTGTCGGCAGCTCT, shCamta2B: GGAGGGGGTGTACATAGGA. For simultaneous expression of two shRNAs a rAAV vector was generated that contains two U6 promoters driving expression of two independent shRNAs. shCtrl2x contains two copies of the nontargeting control shRNA. shCamta $1+2$ contains one copy of shCamta1A and one copy of shCamta $2 B$. rAAV vectors were generated by standard molecular biology techniques and verified by sequencing. Viral particles were produced as described (Zhang et al. 2007).

\section{Stereotaxic surgery}

Virus injection in the dorsal hippocampus was done using the following stereotaxic coordinates: anteroposterior, -2 $\mathrm{mm}$; mediolateral, $\pm 1.5 \mathrm{~mm}$; dorsoventral, $-1.7,-1.9$, and $-2.1 \mathrm{~mm}$ from bregma. A total of $1.5 \mu \mathrm{L}$ of viral solution/ hemisphere was injected. Injections proceeded at a speed of $200 \mathrm{~nL} / \mathrm{min}$ through a $33 \mathrm{G}$ needle. The injection needle was left in place an additional $60 \mathrm{sec}$ to allow the fluid to diffuse. Behavioral or gene expression experiments were performed 3 wk after stereotaxic delivery of rAAVs.

\section{Behavioral studies}

For behavioral experiments we used 3-mo old male C57BL/6NCrl mice. Three days before the memory tests started, all mice were handled $(1 \mathrm{~min}$ per day). Object-place recognition test and Contextual Fear Conditioning were performed as described (Oliveira et al. 2012).

\section{Morphological analyses}

Golgi impregnation was performed using a Rapid Golgi Stain Kit (FD NeuroTechnologies) according to the manufacturer's instructions. Z-stacks of Golgilabeled CA1 neurons were recorded at a $0.9-\mu \mathrm{m}$ interval with a $20 \times$ objective (N.A. 0.75) mounted on a Nikon Eclipse 90i upright microscope (Nikon Imaging Center, University of Heidelberg). For dendritic spine analysis, images of dendritic segments were recorded at a $0.2-\mu \mathrm{m}$ interval with a $100 \times$ objective (N.A. 1.45). Basal dendritic trees and dendritic spines were manually traced using the Simple Neurite Tracer plugin of FIJI software (http://fiji.sc/Fiji). All tracings and analyses were done blind. $P$-values were determined by two-tailed $t$ tests for dendrite length, spine length, and spine density, and repeated-measures two-way ANOVA with Bonferroni post hoc test for Sholl analysis. 
Table 3. Effect of Camta1 knockdown on the electrophysiological properties of CA1 pyramidal neurons in organotypic culture

\begin{tabular}{llcc}
\hline $\begin{array}{l}\text { Membrane } \\
\text { capacitance }\end{array}$ & $\mathrm{pS}$ & $143 \pm 9(26)$ & $142 \pm 13(12)$ \\
$\begin{array}{l}\text { Membrane } \\
\text { resistance }\end{array}$ & $\mathrm{M} \Omega$ & $114 \pm 6(26)$ & $135 \pm 18(12)$ \\
$V_{\text {rest }}$ & $\mathrm{mV}$ & $-57.8 \pm 1.8(23)$ & $-62.2 \pm 1.8(12)^{*}$ \\
AP threshold & $\mathrm{mV}$ & $-30.8 \pm 1.1(23)$ & $-32.4 \pm 1.4(12)$ \\
AP amplitude & $\mathrm{mV}$ & $69.1 \pm 2.1(23)$ & $76.2 \pm 2.4(12)$ \\
AP half width & $\mathrm{msec}$ & $1.47 \pm 0.09(23)$ & $1.52 \pm 0.16(12)$ \\
AHP amplitude & $\mathrm{mV}$ & $-11.9 \pm 0.7(23)$ & $-16.6 \pm 1.4(12)^{* *}$ \\
AHP peak delay & $\mathrm{msec}$ & $18.6 \pm 2.2(23)$ & $23.0 \pm 5.0(12)$ \\
Accommodation & $\mathrm{AU}$ & $0.535 \pm 0.067(23)$ & $0.494 \pm 0.069(12)$ \\
index & & & \\
Inward rectifier & $\mathrm{nS}$ & $3.70 \pm 0.47(23)$ & $2.58 \pm 0.52(12)$ \\
$\quad$ slope & $\mathrm{nS}$ & $7.09 \pm 0.74(23)$ & $8.07 \pm 1.54(12)$ \\
$h$ current slope & $\mathrm{nS}$ & $11.3 \pm 0.8(10)^{*}$ \\
mEPSC amplitude & $\mathrm{pA}$ & $14.1 \pm 0.7(14)$ & $656 \pm 73(10)^{*}$ \\
mEPSC IEI & $\mathrm{msec}$ & $416 \pm 55(14)$ & \\
\hline
\end{tabular}

Parameters were quantified from whole-cell patch clamp recordings in 3-4 wk old cultures after infection with either rAAV-shCtrl or rAAV-shCamta1B.

$\left(V_{\text {rest }}\right)$ resting membrane potential, (AP) action potential, (AHP) afterhyperpolarization potential, (mEPSC) miniature excitatory postsynaptic current, (IEI) inter-event interval.

Data represent mean value \pm standard error of the mean (cell numbers in brackets) of measurements from at least three OTC preparations. $\left(^{*}\right) s \quad P<0.05 ;\left(^{* *}\right) P<0.005$ determined by two-tailed $t$-test.

\section{RNA-seq and data analysis}

For each group (shCtrl, shCamta1A, shCamta1B) three animals were used. All mice were handled ( 1 min per day) for $3 \mathrm{~d}$ before the experiment started. On the experiment day, animals were trained in the object-place recognition test for $30 \mathrm{~min}$ and returned to the home cage for additional $60 \mathrm{~min}$.

We thus focused our analysis on a time point that is known to capture the induction of classical activity-regulated genes (Zhang et al. 2007, 2009). Animals were then killed by cervical dislocation and hippocampi were rapidly isolated. rAAV-infected areas of the dorsal hippocampus were identified by mCherry fluorescence and were rapidly dissected under a microscope and placed in RNAlater (Ambion) until RNA isolation. Since we used the same stereotaxic coordinates for rAAV delivery, the tissue that was used for RNA-seq corresponds to the hippocampal regions that were affected by Camta knockdown in the behavioral experiments. Total RNA was extracted using Qiagen RNeasy mini kit with additional on-column DNase I digestion. Of note, $1.5 \mu \mathrm{g}$ of each total RNA sample was used for RNA-seq. Nonstrand-specific RNA-seq libraries were constructed and sequenced by the CellNetworks Deep Sequencing Core Facility at the University of Heidelberg. Of note, 50-bp paired-end reads in FASTQ format were generated using the Illumina Hiseq2000 platform and aligned to the Mus musculus reference genome (NCBI build 37) using TopHat (Trapnell et al. 2009). Aligned read files (accepted_hits.bam) were name sorted and transformed to SAM files with SAMtools (Li et al. 2009). Raw counts for each gene were obtained with HTSeq-count (Anders et al. 2015) and differential expression was analyzed with the likelihood ratio test using DESeq2 (http://dx.doi.org/10.1101/ 002832). The cutoff was set to a Benjamini-Hochberg adjusted $P$ value of 0.1 . Selected gene sets were analyzed for enrichment of gene ontology (GO) terms and pathways using the bioCompendium database (http://biocompendium.embl.de).

\section{Quantitative reverse transcription PCR}

Total RNA was extracted from rAAV-infected hippocampal tissue using Qiagen RNeasy mini kit with additional on-column DNase I digestion. cDNAs were synthesized from $1 \mu \mathrm{g}$ of total RNA using High Capacity cDNA Reverse Transcription kit (Applied Biosystems). Quantitative reverse transcription PCR (QRT-PCR) was done on an ABI7300 thermal cycler using universal QRT-PCR master mix with the following TaqMan Gene Expression Assays (Applied Biosystems): 18s rRNA (4319413E), Gusb (Mm00446953_m1), Camta1 (Mm01263982_m1), Camta2 (Mm00626346_m1), Arc (Mm00479619.g1), Npas4 (Mm004636 44_m1), Gadd45b (Mm00435123_m1), Fos (Mm00487425_m1), Atp2b4 (Mm01285597_m1), Cnr1 (Mm1212171_s1), Doc2g (Mm01236549_m1), Htr2c (Mm00434127_m1), Kcnd̈3 (Mm0130 2126_m1), Otof (Mm00453306_m1), Scn7a (Mm00801952_m1), Sytl4 (Mm00489110_m1), Tgfb2 (Mm00436955_m1), Trpc7 (Mm00442606_m1). Expression of target genes was normalized against Gusb and/or 18s rRNA as endogenous control genes. For QRT-PCR analysis of CAMTA1 target genes all mice were handled for $3 \mathrm{~d}$ ( 1 min per day) before the experiment started. On the experiment day, one group of animals was left in the home cage (untrained) and one group was trained in the object-place recognition test for $30 \mathrm{~min}$. Animals were sacrificed $60 \mathrm{~min}$ after the end of the training session. For each group (shCtrl, shCamta1A, shCamta1B; basal and trained) six animals were used. shCamta1 infected animals that exhibited $<20 \%$ knockdown compared with shCtrl were excluded from further analysis.
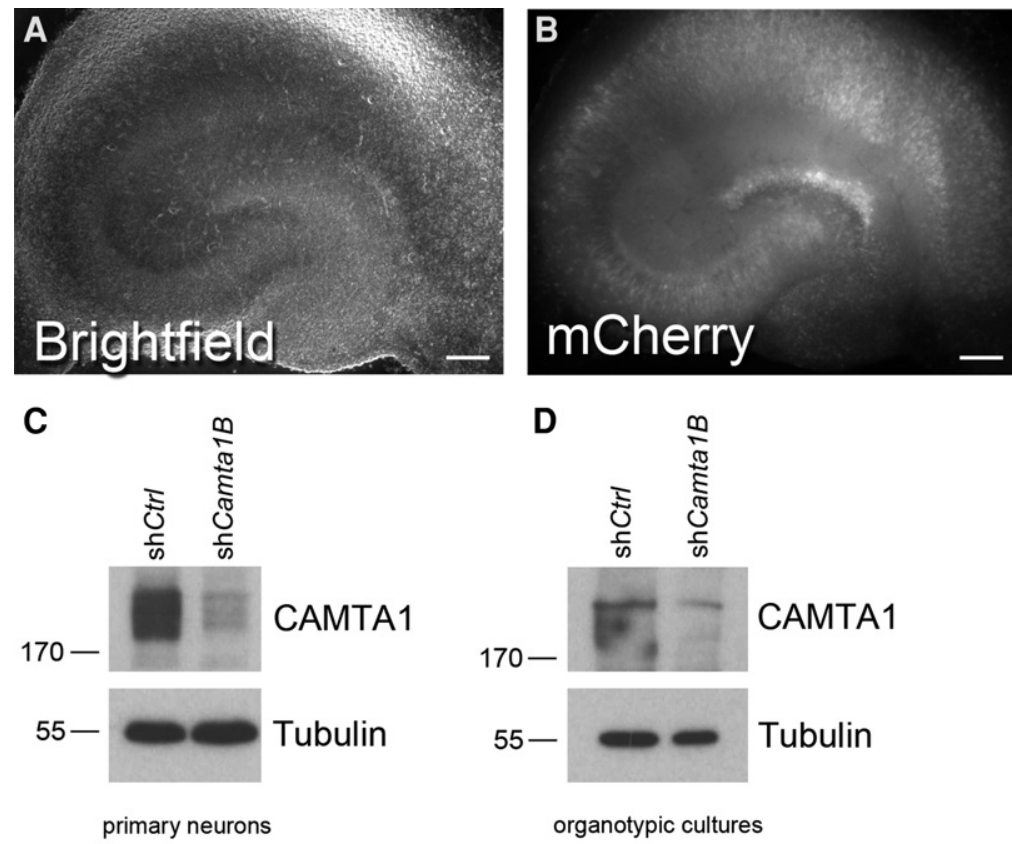

Figure 5. Validation of CAMTA1 knockdown efficiency in rat neurons. Primary rat hippocampal neurons and rat organotypic hippocampal slice cultures were infected with shCtrl or shCamta1. CAMTA1 protein expression was analyzed by Immunoblot. $(A, B)$ Example photomicrographs of an organotypic slice culture infected with rAAV-shCtrl. Scale bar $=200 \mu \mathrm{m}$. $(C, D)$, Representative immunoblots showing expression of CAMTA1 and Tubulin in rat hippocampal neurons (C) and rat organotypic cultures $(D)$. Position of molecular weight markers (kDa) is indicated. 


\section{Whole-cell patch clamp recordings}

Whole-cell patch clamp recordings were made from OTCs at 28 to $35 \mathrm{~d}$ in vitro. Each slice and its underlying membrane was secured with a platinum ring in a recording chamber (OAC-1, Science Products $\mathrm{GmbH}$ ) mounted on a fixed-stage upright microscope (BX51WI, Olympus). Differential interference contrast optics, infrared illumination, and an sCMOS camera (Andor Neo, Acal BFi) were used to view neurons on a computer monitor using a software interface (Andor iQ). Slices were submerged with continuously flowing $(3 \mathrm{~mL} / \mathrm{min})$ artificial cerebrospinal fluid (ACSF, in mM: $\mathrm{NaCl}, 125 ; \mathrm{KCl}, 3.5 ; \mathrm{MgCl}_{2}, 1.3 ; \mathrm{NaH}_{2} \mathrm{PO}_{4}, 1.2 ; \mathrm{CaCl}_{2}, 2.4$; glucose, $25 ; \mathrm{NaHCO}_{3}, 26$; gassed with $95 \% \mathrm{O}_{2}$ and $5 \% \mathrm{CO}_{2}$ ) maintained at $32^{\circ} \mathrm{C}$ with an in-line perfusion heater (TC324B, Warner Instruments Corporation). Patch electrodes (3-4 M $\Omega$ ) were made from $1.5-\mathrm{mm}$ borosilicate glass and filled with internal solution (in $\mathrm{mM}$ : potassium methylsulfate, $133 ; \mathrm{NaCl}, 8 ; \mathrm{KCl}, 12 ; \mathrm{HEPES}$, 10; $\mathrm{K}_{2}$-phosphocreatine, $6 ; \mathrm{Mg}_{2} \mathrm{ATP}, 4 ; \mathrm{Na}_{3} \mathrm{GTP}, 0.5 ; \mathrm{pH} 7.35$ with $\mathrm{KOH})$. Recordings were made with a Multiclamp 700A amplifier, digitized through a Digidata $1322 \mathrm{~A} \mathrm{~A} / \mathrm{D}$ converter and acquired and analyzed using pClamp 10 software (Molecular Devices). Access resistance (range: 5-15 $\mathrm{M} \Omega$ ) was monitored regularly during voltage clamp recordings and data was rejected if changes $>20 \%$ occurred. Membrane potentials have not been corrected for junction potential.

For details of mEPSC acquisition and analysis, see Mauceri et al. 2011. Action potential threshold was determined by the point of maximal inflection. Action potential amplitude and after hyperpolarization were determined relative to the action potential threshold. Accommodation index was calculated as the ratio of the time interval between the first and last pairs of action potentials in response to a 1 sec current injection evoking at least six action potentials. Other action potential parameters were calculated from an action potential evoked by a minimal current injection for $1 \mathrm{sec}$. Inward rectifier slope conductances were calculated from the difference between the linear fits at -60 to $-80 \mathrm{mV}$ and -100 to $-120 \mathrm{mV}$ of the instantaneous current evoked by $1 \mathrm{sec}$ hyperpolarizing steps from a holding potential of $-50 \mathrm{mV}$. From the same current traces, $h$ current slope conductances were calculated as the linear fit of the difference between the instantaneous and steady-state currents from -90 to $-120 \mathrm{mV}$.

\section{Competing interest statement}

None declared.

\section{Acknowledgments}

We thank Iris Bünzli-Ehret for preparation of hippocampal neurons, Ursula Weiss for help with the immunoblot analyses, Daniela Mauceri for providing the rAAV expression vector containing nontargeting control shRNA, Sarina Poehner and Maria Müller for their help with the preparation of organotypic hippocampal cultures, and David Ibberson (CellNetworks Deep Sequencing Core Facility, Heidelberg) for RNA-seq library preparation and advice.

This work was supported by the Deutsche Forschungsgemeinschaft (DFG, BA1007/6-1 to C.B.O. and H.B.), an ERC Advanced Grant (to H.B.), the Excellence Cluster CellNetworks at Heidelberg University, the Sonderforschungsbereich (SFB) 636 of the DFG, and the Graduate Academy (Excellence Initiative) of Heidelberg University, and the German Ministry of Education and Research (BMBF, 01GQ1003A). H.B. is a member of the Excellence Cluster CellNetworks at Heidelberg University.

\section{References}

Alberini CM. 2009. Transcription factors in long-term memory and synaptic plasticity. Physiol Rev 89: 121-145.

Anders S, Pyl PT, Huber W. 2015. HTSeq--a Python framework to work with high-throughput sequencing data. Bioinformatics 31: 166-169.

Bading H. 2013. Nuclear calcium signalling in the regulation of brain function. Nat Rev Neurosci 14: 593-608.

Bading H, Greenberg ME. 1991. Stimulation of protein tyrosine phosphorylation by NMDA receptor activation. Science 253: 912-914.
Bading H, Ginty DD, Greenberg ME. 1993. Regulation of gene expression in hippocampal neurons by distinct calcium signaling pathways. Science 260: $181-186$

Bouché N, Scharlat A, Snedden W, Bouchez D, Fromm H. 2002. A novel family of calmodulin-binding transcription activators in multicellular organisms. J Biol Chem 277: 21851-21861.

Finkler A, Ashery-Padan R, Fromm H. 2007. CAMTAs: calmodulin-binding transcription activators from plants to human. FEBS Lett 581: 3893-3898.

Han J, Gong P, Reddig K, Mitra M, Guo P, Li HS. 2006. The fly CAMTA transcription factor potentiates deactivation of rhodopsin, a G protein-coupled light receptor. Cell 127: 847-858.

Henrich KO, Bauer T, Schulte J, Ehemann V, Deubzer H, Gogolin S, Muth D, Fischer M, Benner A, Konig R, et al. 2011. CAMTA1, a 1p36 tumor suppressor candidate, inhibits growth and activates differentiation programs in neuroblastoma cells. Cancer Res 71: 3142-3151.

Huentelman MJ, Papassotiropoulos A, Craig DW, Hoerndli FJ, Pearson JV, Huynh KD, Corneveaux J, Hanggi J, Mondadori CR, Buchmann A, et al. 2007. Calmodulin-binding transcription activator 1 (CAMTA1) alleles predispose human episodic memory performance. Hum Mol Genet 16: 1469-1477.

Lau D, Bading H. 2009. Synaptic activity-mediated suppression of p53 and induction of nuclear calcium-regulated neuroprotective genes promote survival through inhibition of mitochondrial permeability transition. J Neurosci 29: 4420-4429.

Lein ES, Hawrylycz MJ, Ao N, Ayres M, Bensinger A, Bernard A, Boe AF, Boguski MS, Brockway KS, Byrnes EJ, et al. 2007. Genome-wide atlas of gene expression in the adult mouse brain. Nature 445: 168-176.

Li H, Handsaker B, Wysoker A, Fennell T, Ruan J, Homer N, Marth G, Abecasis G, Durbin R; 1000 Genome Project Data Processing Subgroup. 2009. The Sequence Alignment/Map format and SAMtools. Bioinformatics 25: 2078-2079.

Limback-Stokin K, Korzus E, Nagaoka-Yasuda R, Mayford M. 2004. Nuclear calcium/calmodulin regulates memory consolidation. J Neurosci 24: $10858-10867$.

Long C, Grueter CE, Song K, Qin S, Qi X, Kong YM, Shelton JM, Richardson JA, Zhang CL, Bassel-Duby R, et al. 2014. Ataxia and Purkinje cell degeneration in mice lacking the CAMTA1 transcription factor. Proc Natl Acad Sci 111: 11521-11526.

Mauceri D, Freitag HE, Oliveira AM, Bengtson CP, Bading H. 2011. Nuclear calcium-VEGFD signaling controls maintenance of dendrite arborization necessary for memory formation. Neuron 71: 117-130.

Mellstrom B, Savignac M, Gomez-Villafuertes R, Naranjo JR. 2008. $\mathrm{Ca}^{2+}$-operated transcriptional networks: molecular mechanisms and in vivo models. Physiol Rev 88: 421-449.

Miller LA, Gunstad J, Spitznagel MB, McCaffery J, McGeary J, Poppas A, Paul RH, Sweet LH, Cohen RA. 2011. CAMTA1 T polymorphism is associated with neuropsychological test performance in older adults with cardiovascular disease. Psychogeriatrics 11: $135-140$.

Oliveira AM, Hemstedt TJ, Bading H. 2012. Rescue of aging-associated decline in Dnmt3a2 expression restores cognitive abilities. Nat Neurosci 15: $1111-1113$.

Song K, Backs J, McAnally J, Qi X, Gerard RD, Richardson JA, Hill JA Bassel-Duby R, Olson EN. 2006. The transcriptional coactivator CAMTA2 stimulates cardiac growth by opposing class II histone deacetylases. Cell 125: 453-466.

Stoppini L, Buchs PA, Muller D. 1991. A simple method for organotypic cultures of nervous tissue. J Neurosci Methods 37: 173-182.

Thevenon J, Lopez E, Keren B, Heron D, Mignot C, Altuzarra C Beri-Dexheimer M, Bonnet C, Magnin E, Burglen L, et al. 2012. Intragenic CAMTA1 rearrangements cause non-progressive congenital ataxia with or without intellectual disability. J Med Genet 49: 400-408.

Trapnell C, Pachter L, Salzberg SL. 2009. TopHat: discovering splice junctions with RNA-Seq. Bioinformatics 25: 1105-1111.

Uhlen M, Oksvold P, Fagerberg L, Lundberg E, Jonasson K, Forsberg M, Zwahlen M, Kampf C, Wester K, Hober S, et al. 2010. Towards a knowledge-based Human Protein Atlas. Nat Biotechnol 28: 1248-1250.

West AE, Greenberg ME. 2011. Neuronal activity-regulated gene transcription in synapse development and cognitive function. Cold Spring Harb Perspect Biol 3. doi: 10.1101/cshperspect.a005744.

Yang T, Poovaiah BW. 2002. A calmodulin-binding/CGCG box DNA-binding protein family involved in multiple signaling pathways in plants. J Biol Chem 277: 45049-45058.

Zhang SJ, Steijaert MN, Lau D, Schutz G, Delucinge-Vivier C, Descombes P, Bading H. 2007. Decoding NMDA receptor signaling: identification of genomic programs specifying neuronal survival and death. Neuron 53: $549-562$

Zhang SJ, Zou M, Lu L, Lau D, Ditzel DA, Delucinge-Vivier C, Aso Y, Descombes P, Bading H. 2009. Nuclear calcium signaling controls expression of a large gene pool: identification of a gene program for acquired neuroprotection induced by synaptic activity. PLoS Genet 5: e1000604.

Received November 14, 2015; accepted in revised form April 4, 2016. 


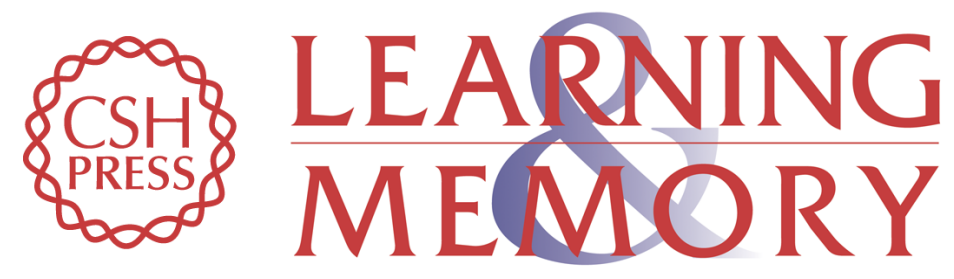

\section{The calmodulin-binding transcription activator CAMTA1 is required for long-term memory formation in mice}

Carlos Bas-Orth, Yan-Wei Tan, Ana M.M. Oliveira, et al.

Learn. Mem. 2016, 23:

Access the most recent version at doi:10.1101/Im.041111.115

\section{Supplemental http://learnmem.cshlp.org/content/suppl/2016/05/11/23.6.313.DC1 Material}

References This article cites 28 articles, 10 of which can be accessed free at: http://learnmem.cshlp.org/content/23/6/313.full.html\#ref-list-1

Creative This article is distributed exclusively by Cold Spring Harbor Laboratory Press for the Commons first 12 months after the full-issue publication date (see

License http://learnmem.cshlp.org/site/misc/terms.xhtml). After 12 months, it is available under a Creative Commons License (Attribution-NonCommercial 4.0 International), as described at http://creativecommons.org/licenses/by-nc/4.0/.

Email Alerting Receive free email alerts when new articles cite this article - sign up in the box at the Service top right corner of the article or click here. 